\section{Letter: Preliminary National Survey Results Evaluating the Impact of COVID-19 Pandemic on Medical Students Pursuing Careers in Neurosurgery}

To the Editor:

The COVID-19 pandemic represents "unprecedented times" for all, including those involved in medical student education. ${ }^{1}$ Currently, attention has been appropriately focused on providing patient care and educating medical professionals on the evolving understanding of and treatment recommendations for patients suffering from COVID-19. These urgent clinical needs have led to profound disruption of neurosurgical practices, the magnitude of which in America is unclear, but international survey data allude to the significance. ${ }^{2}$ Efforts to minimize collateral damage to neurosurgical student education have received relatively less attention. Medical students with intentions of pursuing careers in neurosurgery or transitioning to internship are a vulnerable population that may be disconnected and lacking critical mentorship, clinical exposure, and formal relationships to local and national neurosurgical communities. On March 17, 2020, the Association of American Medical Colleges issued guidelines that supported a pause in clinical rotations for medical students. ${ }^{3}$ After the cancellation of clinical rotations, medical students have remained engaged by supporting the hospital community through collecting masks, staffing calls centers, creating patient education materials, and offering childcare service to frontline health care workers. ${ }^{4}$ The pandemic has significantly disrupted educational opportunities, and experiences for the youngest physicians in training, but its impact remains unclear.

In this report, we summarize the preliminary findings from a national survey that assessed the impact of the COVID-19 pandemic on medical students considering or already transitioning to neurosurgical careers. We conducted a cross-sectional, anonymous online survey distributed to medical students enrolled in and foreign medical graduates working at United States medical schools. We contacted survey respondents through publicly available email addresses, interest groups, and social media threads (Twitter and Facebook), and here we describe preliminary findings from 7 days of data collection.

A total of 315 respondents completed the survey. Using data from the most recent senior applicants in this year's 2020 Match, we estimate a preliminary response rate among third-year medical students (MS3s) of approximately 36\%. Approximately onethird of respondents were MS3s, and the majority were male sex in their late 20 s, and nearly $20 \%$ from schools without home neurosurgical departments. Across all respondents, approximately two-thirds have reported indefinite postponement of clinical clerkship and most have suspended in-person didactics. Many reported using unstructured time to improve neurosurgical knowledge, with increasing frequency by increasing medical school years. More than half of the participants report that the pandemic has had a significant negative impact on academic productivity. The pandemic is affecting all levels of student education. A total of 1 in 3 MS1s reported dissatisfaction with neurosurgical career planning offered by their home medical school, and almost 1 in 5 stated they are now less likely to pursue a career in neurosurgery. The majority of MS2s and MS3s are delaying their United States Medical Licensing Examination Steps I and II. Among MS3s, at least 3 in 4 reported indefinite postponement of subinternships, and most are unsatisfied with communication from external programs as it relates to subinternships. Over one-third of MS4s are graduating early to participate in COVID-19-related patient care and the vast majority are requesting logistical help to prepare for residency remotely. When asked how the neurosurgical community can better support students during the pandemic, the most popular requests were student-focused webinars, student-focused sessions at upcoming neurosurgical conferences, and finding ways to accommodate for expected changes in external subinternships, if possible.

The future of neurosurgery relies critically on our recruitment and retention of talented young physicians. The pandemic is clearly impacting every aspect in clinical neurosurgical practice, and its ramification on medical education from the youngest generations to seasoned neurosurgeons is yet to be fully understood. Medical students pursuing careers in neurosurgery have unique academic, career, and personal challenges that serve as unique opportunities to support and engage some of the most motivated students in medical training. As the neurosurgical community navigates the pandemic, we hope these findings will help support students' interests, and provide an additional framework to further identify creative solutions to engage those needing support. As we review the results of the preliminary data collection, we have identified several key actionable items that could potentially help support and foster relationships with MSs more holistically. These ideas include:

- Offer students additional virtual educational opportunities, including invitations to departmental conferences and national conference activities.

- Provide virtual opportunities for mentorship, on a one-to-one or small group basis.

- Engage students to participate in COVID-19 era research.

- Create virtual programs for neurosurgery interest groups.

- Mitigate the impact of the lack of away rotations for MS3s, and its impact on letters of recommendation, particularly among students without home neurosurgery programs.

- Support MS4s in anticipation of starting intern year without "boot camp."

- Offer remote housing and logistical resources to recently matched MS4s. 
- Extend invitations to online academic neurosurgical webinars, and tailor topics specifically for students.

As we prepare for the unpredictable future within the COVID19 pandemic, we must safeguard and ensure that the future of neurosurgery remains strong and able to carry forth the spirit of leadership and innovation that is central to our community. These are truly unprecedented times, and as we continue to gather more information, we hope to share these results with the neurosurgical community in a timely fashion. We hope that the data will continue to ignite creative solutions to unite all ages of neurosurgeons in training and practice.

\section{Disclosures}

Dr Reynolds serves as a Fogarty Global Health Trainee affiliated with the Vanderbilt-Emory-Cornell-Duke consortium and is supported by the Fogarty International Center at the National Institutes of Health under grant number R25TW009337. Dr Garcia serves as a Fogarty Global Health Trainee and is supported by the Fogarty International Center under grant number D43TW010543 through the Harvard, Boston University, Northwestern University, and University of New Mexico consortium. The content is solely the responsibility of the authors and does not necessarily represent the official views of the National Institutes of Health. The authors have no personal, financial, or institutional interest in any of the drugs, materials, or devices described in this article.

\author{
${ }^{S}$ Department of Neurosurgery \\ Vanderbilt University Medical Center \\ Nashville, Tennessee \\ 'Ann and Robert H. Lurie Children's Hospital \\ Chicago, Illinois \\ "Department of Neurosurgery \\ George Washington University School of Medicine and Health \\ Sciences \\ Washington, District of Columbia
}

\section{REFERENCES}

1. Rose S. Medical student education in the time of COVID-19. JAMA. published online: March 31, 2020 (doi:10.1001/jama.2020.5227).

2. Jean WC, Ironside NT, Sack KD, Felbaum DR, Syed HR. The impact of COVID-19 on neurosurgeons and the strategy for triaging non-emergent operations: a global neurosurgery study. Acta Neurochir. published online: April 21, 2020 (doi:10.1007/s00701-020-04342-5).

3. Association of American Medical Colleges. Guidance on Medical Students' Participation in Direct Patient Contact Activities, April 14, 2020. https://www. aamc.org/system/files/2020-04/meded-April-14-Guidance-on-Medical-StudentsParticipation-in-Direct-Patient-Contact-Activities.pdf. Accessed April 21, 2020.

4. Krieger P, Goodnough A. Medical students, sidelined for now, find new ways to fight coronavirus. The New York Times. March 23, 2020. https://www.nytimes. com/2020/03/23/health/medical-students-coronavirus.html. Accessed April 21, 2020.

5. National Resident Matching Program. Advance Data Tables 2020 Main Residency Match. https://mk0nrmp3oyqui6wqfm.kinstacdn.com/wp-content/uploads/2020/ 03/Advance-Data-Tables-2020.pdf. Accessed April 23, 2020.

\section{Acknowledgments}

We gratefully acknowledge all the medical students who participated in the piloting and creation of this survey from George Washington University, Northwestern University, Tufts University, and Vanderbilt University. Your rapid responses were essential to the timely realization of this manuscript.

Copyright $(2020$ by the Congress of Neurological Surgeons

10.1093/neuros/nyaa214 